\title{
A Novel Method to Design Stub-Loaded Microstrip Filters with Arbitrary Passband Based on the Reflection Theory and Monte Carlo Method
}

\author{
Yuhong MA, Ye YUAN, Wentao YUAN, Weiwei WU, Naichang YUAN \\ State Key Laboratory of Complex Electromagnetic Environment Effects on Electronics and Information System, \\ National University of Defense Technology, Changsha, Co 410073 China \\ yuanyeaheng@icloud.com
}

Submitted July 14, 2018 / Accepted December 30, 2018

\begin{abstract}
In this paper, a novel method to design microstrip filters with arbitrary passband is proposed. A kind of stubloaded microstrip filter with asymmetric structure is proposed in this paper. The mathematic function of the total reflection coefficient at the input port of the filter is derived based on the reflection theory, and an ultra-wide-band (UWB) filter which can work from $11 \mathrm{GHz}$ to $39 \mathrm{GHz}$ is fabricated and measured to verify that our function can calculate the reflection coefficient precisely. The circuit model of this UWB filter is provided, and the comparison between frequency responses of the circuit simulation and the calculation results justifies the validity of our proposed method. The procedure based on the Monte Carlo method to design stub-loaded microstrip filters with arbitrary passbands is given in this paper. A bandpass filter (BPF) which can work on 7-9.5 GHz is designed and measured as an example. The comparison between calculated dimensions and practical dimensions of this filter shows a satisfactory fitting degree. Then the proposed method is compared with the Chebyshev filter design method to show the advantages of our proposed method. A low-pass filter (LPF) which can work below $4 \mathrm{GHz}$ is designed and compared with some prior arts in order to show that our proposed filter is simple in structure and small in size. At last, the adaptabilities of our proposed method in narrow band and multi-band situations are discussed in this paper. By using our proposed method, the stub-loaded microstrip filter can be designed quickly and easily. Based on the results mentioned above, we can count that the method proposed in this paper has a good application in microstrip filter design.
\end{abstract}

\section{Keywords}

Electromagnetic reflection, microstrip filters, Monte Carlo methods, reflection coefficient

\section{Introduction}

\subsection{Motivation and Related Works}

Microstrip filters have been widely used in a large number of RF applications. As the wireless communication systems are developing rapidly today, it is important for RF engineers to hunt for some convenient methods to design microstrip filters [1].

A general design method for multiband filters is proposed in [2]. In [2], the frequency and element transformations from a low-pass prototype filter to a practical multiband bandpass filter is derived, and the formula which extracts the coupling coefficient $k$ between coupled multimode resonators and the external quality factor are obtained. A novel method to design low loss dual-band microstrip filters using folded open-loop ring resonators (OLRRs) is presented in [3]. The first and second passbands of the dual-band filter can be adjusted to a desired frequency bands by adjusting the physical dimensions of OLRRs. A novel microstrip Chebyshev low-pass filter (LPF) design method using a defected ground structure (DGS) is introduced in [4]. The DGS reduces the lengths of inductive microstrip lines effectively, so the miniaturization of the filter dimensions is achieved. In this paper, a novel method to design stub-loaded microstrip filters is proposed. The whole design procedure is based on the reflection theory and Monte Carlo method.

In [5], the theory of small reflections (TSR) and its derivation process is introduced. The TSR is to consider the total reflection coefficient at the input port as the composite function of local reflection coefficients between the neighboring transmission lines [5], [6]. This theory is widely used in the design of impedance matching between unmatched sources and loads [7-11]. In [9], a microstrip feeding for an antenna is designed based on the TSR, and the return loss in its working frequency band is under $-60 \mathrm{~dB}$. This result verifies that the TSR has good guidance in impedance matching design. 
Stepped-impedance filters also apply the TSR to design some well performed low-pass filters (LPFs) [12-17]. In [17], the authors generalize this theory for the case of multi-section transformers. In [24], a LPF which can work below $4 \mathrm{GHz}$ is designed based on the stepped-impedance microstrip lines, and it is an example for the traditional design procedure of stepped-impedance microstrip LPFs.

The filters applying stepped-impedance resonators are studied in recent years [18-23]. A microstrip low-pass filter (LPF) with three coupled stepped impedance resonators is presented in [21]. This filter has wide stopband bandwidth and can offer a high selectivity. In [22], a widestopband bandpass filter based on silted stepped-impedance resonators is introduced. Using silted stepped-impedance resonators can widen the bandwidth of the stop-band of the filter and add more freedoms in controlling resonance modes compared with a stepped-impedance resonator. Steppedimpedance ring resonator is also used to construct balanced tri-band bandpass filters which have excellent out-of-band performance [23].

However, there is a limitation while using the TSR to design microstrip filters: if the impedance between two neighboring stubs has a large discrepancy, some approximations based on the TSR will be incorrect. In [25], the authors present a modified version of TSR and use this technology to design a broadband filter, but the frequency response of this filter dose not perform well.

In [26], we propose a method based on the TSR to design microstrip filters with arbitrary passbands. In that paper, a novel stub-loaded microstrip filter is proposed and the approximative reflection coefficient function at the input port of the filter is derived. In this paper, we present a modified version of the method in [26]. Our modified method can achieve more accurate calculation results than the method in [26]. The reflection coefficient function becomes very complex, so it is difficult for us to solve the dimensions of our filters from the reflection coefficient function. Therefore, it is important for us to find some feasible calculation methods to figure out this problem.

In this paper, we choose the Monte Carlo method as our reverse calculation method. The Monte Carlo method is an efficient mathematical tool to solve some extremely complicated problems [27]. In [28], the authors propose a transition between a microstrip line and a ridge gap waveguide, and they use the Monte Carlo method to analysis the assembly tolerances.

By using our modified method, the design procedure is simplified and the proposed microstrip filter can be designed simply and quickly compared with other methods.

\subsection{Summary of the Contributions and Paper Organization}

The main contributions of our present study are summarized as follows:

- A novel multiple-stub-loaded impedance transformer filter is proposed in this paper. We use the reflection theory to calculate the total reflection coefficient of this types of filter and derive the accurate reflection function at the input port. To verify that our proposed function works well, an UWB filter is simulated and fabricated, and the calculation results of $S$-parameter fit well with the practical measurement results. Also, the circuit model of this UWB filter is given, and the frequency responses of the circuit simulation are compared with the calculation results of the proposed method. The comparison results verify that our proposed method can work as good as the circuit method.

- The proposed reflection function is used to calculate the practical dimensions of our filter. To achieve this purpose, the Monte Carlo method is used as the reverse solution method to find the optimal approximation of our design goals. The relationship between the number of Monte Carlo experiments and the fitting error is investigated. The whole procedure is given in the paper and a BPF is designed by our method to verify the feasibility of our proposed method. In Sec. 3, the proposed BPF is redesigned by the Chebyshev method. The design procedure of the Chebyshev method is compared with our proposed calculation procedure, and the results show that our method can obtain the dimensions of the filter simply and directly.

- In Sec. 3, the comparisons between our proposed method and other prior arts are shown, and the characteristics of our proposed method are discussed. A LPF is designed and compared with filters proposed in [24] and [29] in order to show the advantages of our proposed method and filter. At last, the frequency responses of a dual-band microstrip filter proposed in [26] is recalculated by the method proposed in this paper to justify the application of out modified method in the narrow band and multi-band situations.

The rest of this paper is organized as follows. Section 2 shows our theory and contains the derivation of the total reflection coefficient of multiple-stub-loaded filters at the input port. Section 3 shows the design procedure which bases on the Monte Carlo experiments, and the advantages of our proposed method are also discussed in Sec. 3. Finally, we conclude our paper in Sec. 4.

There are lots of notations used in this paper and they are summarized in Tab. 1. 


\begin{tabular}{|c|c|}
\hline Notation & Implication \\
\hline$\Gamma_{\text {total }}$ & total reflection coefficient \\
\hline$\Gamma_{n}$ & the $n$th local reflection coefficient \\
\hline$Y_{0}$ & $\begin{array}{l}\text { characteristic admittance of the main transmis- } \\
\text { sion line }\end{array}$ \\
\hline$Y_{\mathrm{Im}}^{(n)}$ & $\begin{array}{l}\text { the } n \text {th imaginary of the characteristic admit- } \\
\text { tance }\end{array}$ \\
\hline$Y_{\mathrm{L}}$ & equivalent admittance of the loaded stubs \\
\hline$Y_{x}$ & $\begin{array}{l}\text { equivalent admittance of opened-stub or } \\
\text { shorted-stub }\end{array}$ \\
\hline$N$ & order of filter \\
\hline$f$ & frequency \\
\hline$f_{n}$ & the $n$th sampling point of frequency \\
\hline$L^{(n)}$ & length of the $n$th main transmission line \\
\hline$L_{x}^{(n)}$ & length of the $n$th loaded stub \\
\hline$W$ & width of the microstrip line \\
\hline$\theta_{x}^{(n)}$ & $\begin{array}{l}\text { equivalent electrical length of the } n \text {th loaded } \\
\text { stub }\end{array}$ \\
\hline$\left|S_{11}\right|$ & return loss \\
\hline$\left|\mathbf{S}_{11}\right|$ & vector form of $\left|S_{11}\right|$ \\
\hline$\left|S_{21}\right|$ & insert loss \\
\hline$\left|\mathbf{S}_{21}\right|$ & vector form of $\left|S_{21}\right|$ \\
\hline$F_{\text {goal }}$ & optimization goals \\
\hline $\mathbf{F}_{\text {goal }}$ & vector form of $F_{\text {goal }}$ \\
\hline$M$ & number of Monte Carlo experiments \\
\hline$\varepsilon$ & objection function \\
\hline$\varepsilon_{\text {pass }}$ & objection function in the passband \\
\hline$\varepsilon_{\text {stop }}$ & objection function in the stop-band \\
\hline$\varepsilon_{\min }$ & the minimum value of $\varepsilon$ \\
\hline$x^{(n)}$ & flag bit of the $n$th stub \\
\hline$f_{\mathrm{c}}^{(\text {pass })}$ & $3 \mathrm{~dB}$ passband cut-off frequency \\
\hline$f_{\mathrm{c}}^{(\text {stop })}$ & $20 \mathrm{~dB}$ stop-band cut-off frequency \\
\hline
\end{tabular}

Tab. 1. Notations used in this paper.

\section{Theory Background}

\subsection{Problem Statement}

The [5] shows the method which is used to obtain the total reflection coefficient $\left|\Gamma_{\text {total }}\right|$ of the multiple-stub impedance transformer at the input port. This method is known as the TSR. In [26], a modified version of this theory is proposed and implemented in microstrip filter design, and a novel kind of stub-loaded filters is proposed. The parallel opened- and shorted-stubs are used to change the norm of the local reflection coefficients of the main transmission line in this novel stub-loaded filter. (The detail of this operation is explained in (5)). By using this method, the reflection curve can be shaped in some more complex forms [26]. The equivalent model of this type of filter is shown in Fig. 1. Where $L$ represents the length of each part of the main transmission line, $L_{x}^{(n)}$ represents the dimension of each parallel loaded-stub, $N$ represents the orders of the filter, and $\Gamma_{n}$ represents the local reflection coefficient of neighboring parts.
The $\left|\Gamma_{\text {total }}\right|$ function is shown in (3), where $f$ represents the frequency:

$$
\begin{gathered}
Y_{n}=Y_{0}+Y_{\mathrm{Im}}^{(n)}, \\
\Gamma_{n}(f)=\frac{Y_{n}\left(L_{x}^{(n)}, f\right)-Y_{n+1}\left(L_{x}^{(n+1)}, f\right)}{Y_{n}\left(L_{x}^{(n)}, f\right)+Y_{n+1}\left(L_{x}^{(n+1)}, f\right)}, \\
\left|\Gamma_{\text {total }}(f)\right|=\sum_{N}\left|\Gamma_{n}(f)\right| \exp \left(-\mathrm{j} \frac{4 \pi n L}{\mathrm{c}} f\right) .
\end{gathered}
$$

In [26], equation (3) is used to design the frequency response of the filter. Two microstrip filters are designed and measured. The measured results fit well with the calculated results. But there are some limits while using the method proposed in [26]:

- Equation (3) is based on the TSR, and it is an approximation formula of the practical $\left|\Gamma_{\text {total }}\right|$ function. When the characteristic impedances between the neighbouring parts of the filter differ sharply, this theory will not be applicable. Therefore, the calculation results of the $\left|\Gamma_{\text {total }}\right|$ curve can only indicate the tendency of the pratical $\left|\Gamma_{\text {total }}\right|$ curve but cannot calculate the curve precisely.

- Equation (3) is simplified and shown in (4) while the structure of the filter is chosen to use the centrosymmetric structure.

$$
\begin{aligned}
\Gamma_{\text {total }}(f)= & 2 \mathrm{j} \exp \left(-\mathrm{j} \frac{24 \pi L}{\mathrm{c}} f\right) \times \\
& \sum_{N} \Gamma_{n}\left(L_{n}, L_{n+1}, f\right) \sin \left(\frac{4 \pi n L}{\mathrm{c}} f\right) .
\end{aligned}
$$

This means that a pair of symmetric stubs can only make contributions to one $\left|\Gamma_{n}\right|$, and it will lead to a waste of orders of the filter (the reason will be discussed in Sec. 3.1.1).

- In [26], the gradient descent method is used to optimize (4) (making (4) approximates the objection reflection function). By using the design procedure based on this method, the dimensions of the filter can be predicted. However, (4) is a non-convex function. Therefore, the optimized results are highly related to the initial iterative values [30], and the optimization procedure is time-consuming problem.

To avoid these shortages, this paper proposes a modified method to design a kind of asymmetry stub-loaded microstrip filters.

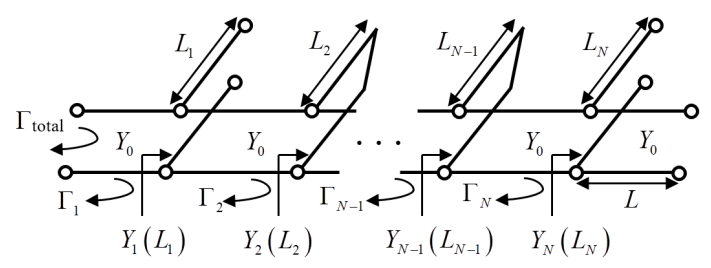

Fig. 1. The equivalent model of microstrip filters proposed in [26]. 


\subsection{Reflection Theory}

Firstly, we want to show the calculation process of $\left|\Gamma_{\text {total }}\right|$ of the single-stub impedance transformer loaded with one opened- or shorted-stub. The equivalent model is shown in Fig. 2, where $Y_{\mathrm{L}}$ represents the equivalent admittance of the load, $\theta$ represents the equivalent electrical length of the main transmission line, $\theta_{x}$ represents the equivalent electrical length of the loaded stub, $\Gamma$ represents the reflection coefficient at the input port, and $Y_{x}$ depends on whether the loaded stub is an opened-stub or a shorted-stub.

If the $Y_{\mathrm{L}}, Y_{1}$ and $Y_{2}$ are set to be equivalent to the same value $Y_{0}$, which means the main transmission line and the load $L$ are matched with each other, the loaded stub and the main transmission line can be regarded as a parallel combination at point $A$. The equivalent admittances $Y_{A}$ of the opened-stub and the shorted-stub loaded situations are shown in (5), respectively:

$$
\left\{\begin{array}{l}
Y_{A}^{\text {(opened) }}=Y_{0}+\mathrm{j} Y_{0} \tan \theta_{x}, \text { while } Y_{x}=0, \\
Y_{A}^{\text {(shorted })}=Y_{0}-\mathrm{j} Y_{0} \cot \theta_{x}, \text { while } Y_{x}=\infty .
\end{array}\right.
$$

For $\Gamma_{1}$, if we assume that the characteristic impedances of all transmission lines of the multi-section structure equal to each other, the opened-stub loaded and shorted-stub loaded situations are shown as follows [26]:

$$
\begin{aligned}
& \Gamma_{1}^{(\text {opened })}=\frac{-\mathrm{j}}{\mathrm{j}+2 \cot \theta_{x}}, \\
& \Gamma_{1}^{\text {(shorted })}=\frac{\mathrm{j}}{2 \tan \theta_{x}-\mathrm{j}} .
\end{aligned}
$$

Then the $\Gamma$ can be derived as (8):

$$
\Gamma=\frac{\left|\Gamma_{1}\right|+\left|\Gamma_{2}\right| \exp (-2 \mathrm{j} \theta)}{1+\left|\Gamma_{1}\right|\left|\Gamma_{2}\right| \exp (-2 \mathrm{j} \theta)}
$$

In [26], we assume that the value of $\left|\Gamma_{1}\right|\left|\Gamma_{2}\right|$ is much smaller than 1, therefore, the denominator in (8) can be ignored. In this paper, we use (8) rather than its approximate form to deal with large local reflected situation and derive the more accurate $\left|\Gamma_{\text {total }}\right|$ function.

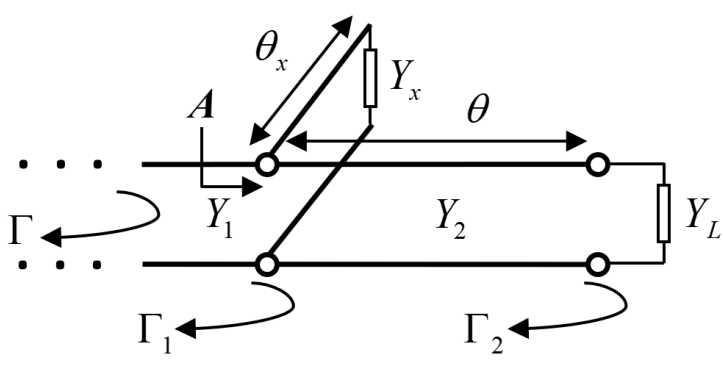

Fig. 2. The equivalent model of single-stub impedance transformer loaded with one opened- or shorted-stub.
The structure shown in Fig. 1 can be decomposed into $N$ parts of the structure which is shown in Fig. 2. From $N$ th part to the 1st part, we can finally derive the $\left|\Gamma_{\text {total }}\right|$ function by calculating $N$ times. The derivation steps of $\left|\Gamma_{\text {total }}\right|$ from (8) are shown in (9), where $\Gamma^{(i)}, \Gamma_{1}^{(i)}$ and $\Gamma_{2}^{(i)}$ represents the corresponding reflection coefficient of the $i$ th part.

$$
\begin{aligned}
& \Gamma^{(N)}=\left|\Gamma_{1}^{(N)}\right|, \text { while } \Gamma_{2}^{(N)}=0 \\
& \Downarrow \\
& \Gamma^{(N-1)}=\frac{\left|\Gamma_{1}^{(N-1)}\right|+\left|\Gamma^{(N)}\right| \exp (-2 \mathrm{j} \theta)}{1+\left|\Gamma_{1}^{(N-1)}\right|\left|\Gamma^{(N)}\right| \exp (-2 \mathrm{j} \theta)} \\
& \Downarrow \\
& \vdots \\
& \Downarrow \\
& \Gamma_{\text {total }}=\Gamma^{(1)}=\frac{\left|\Gamma_{1}^{(1)}\right|+\left|\Gamma^{(2)}\right| \exp (-2 \mathrm{j} \theta)}{1+\left|\Gamma_{1}^{(1)}\right|\left|\Gamma^{(2)}\right| \exp (-2 \mathrm{j} \theta)} .
\end{aligned}
$$

By the $\Gamma_{\text {total }}$ function, the dimensions of the stubs can be obtained directly, and this will be discussed in Sec. 3.1. To verify the feasibility of this modified calculation method, we build a fifth-order shorted-stub loaded microstrip structure (shown in Fig. 3) in the EM simulation software.

All parts of the main line use the same length $L$ and width $W$, as well as the stubs (length $L_{x}$ and width $W$ ). The shorted-stubs is achieved by placing via holes at the end of opened-stubs, and the dielectric substrate is chosen to be Rogers RO4350 (dielectric constant 3.66). The dimensions of this structure are shown in Tab. 2.

The calculation results and simulation results are shown in Fig. 4, where $\left|S_{11}\right|$ represents the return loss and $\left|S_{21}\right|$ represents the insert loss.

\begin{tabular}{|l|l|l|l|}
\hline & $L$ & $L_{x}$ & $W$ \\
\hline $\begin{array}{l}\text { Dimension } \\
{[\mathrm{mm}]}\end{array}$ & 3 & 1.6 & 0.5 \\
\hline
\end{tabular}

Tab. 2. Dimensions of the structure shown in Fig. 3.

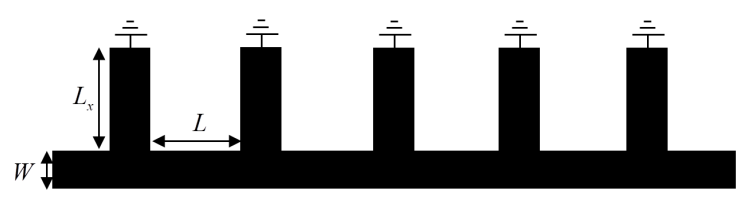

Fig. 3. Fifth-order shorted-stub loaded microstrip structure. 
Figure 4 (a) is the calculation results based on the method in [26] (shown in (4)). We can observe that the calculated result obtained by (4) cannot matching the practical $\left|\Gamma_{\text {total }}\right|$ curve according to Fig. 4 (a), especially with in the stop-band. Fig. 4 (b) illustrates that the modified method in this paper can obtain a quite accurate calculation result. The reason of this improvement is shown just after (8), but to achieve this improvement, the cost is also obvious: the $\left|\Gamma_{\text {total }}\right|$ function will be an extremely complex formula (9), and it is difficult to calculate the dimensions of the filter by inverse solution through (9).

The equivalent circuit model of the structure shown in Fig. 3 is discussed. For shorted-stub, the rectangular metal line is modeled as $L_{1}$ and the coupling between the metal line and the ground introduce the capacitor $C_{1}$. So every shortedstub is modeled as the parallel LC resonator. Every part of the main line is modeled as the cascade of $C_{0}$ and $L_{0}$. The equivalent circuit is shown in Fig. 5. The values of lumped components are optimized in EM software. The equivalent circuit parameters are shown in Tab. 3 .

The frequency responses of the circuit simulation are shown in Fig. 6. Compared with the calculated frequency responses obtained by our proposed method, we can find that the frequency responses of the equivalent circuit simulation result fits well with the curves obtained by (9).

At the end of this subsection, we want to show the measurement results of the optimized filter based on the structure shown in Fig. 3. The fabricated filter is shown in Fig. 7, and the practical dimensions of this filter are shown in Tab. 4. The results of the measured $S$-parameter are shown in Fig. 8.

As depicted in Fig. 8, this filter has an obvious passband from $11 \mathrm{GHz}$ to $39 \mathrm{GHz}$ ( $3 \mathrm{~dB}$ bandwidth). The return loss in the passband of this filter is lower than $-10 \mathrm{~dB}$, and the out-of-band rejection declines rapidly. The center frequency is around $25 \mathrm{GHz}$ and the relative bandwidth is $112 \%$, therefore, it is an UWB filter.

\begin{tabular}{|l|l|l|l|l|}
\hline $\begin{array}{l}\mathrm{ECM} \\
\text { Parameter }\end{array}$ & $L_{0}(\mathrm{nH})$ & $C_{0}(\mathrm{pF})$ & $L_{1}(\mathrm{nH})$ & $C_{1}(\mathrm{pF})$ \\
\hline value & 0.354 & 0.174 & 0.2 & 0.3 \\
\hline
\end{tabular}

Tab. 3. The equivalent circuit parameters of the filter.

\begin{tabular}{|l|l|l|l|l|l|}
\hline Parameter & $W^{(0)}$ & $W^{(1)}$ & $W^{(2)}$ & $W^{(3)}$ & $W^{(4)}$ \\
\hline $\begin{array}{l}\text { Dimension } \\
{[\mathrm{mm}]}\end{array}$ & 0.6 & 0.55 & 0.45 & 0.45 & 0.55 \\
\hline Parameter & $W_{x}^{(1)}$ & $W_{x}^{(2)}$ & $W_{x}^{(3)}$ & $W_{x}^{(4)}$ & $W_{x}^{(5)}$ \\
\hline $\begin{array}{l}\text { Dimension } \\
{[\mathrm{mm}]}\end{array}$ & 0.4 & 0.7 & 0.6 & 0.7 & 0.4 \\
\hline Parameter & $L$ & $L_{x}$ & & & \\
\hline $\begin{array}{l}\text { Dimension } \\
{[\mathrm{mm}]}\end{array}$ & 3 & 1.6 & & & \\
\hline
\end{tabular}

Tab. 4. Dimensions of the filter.

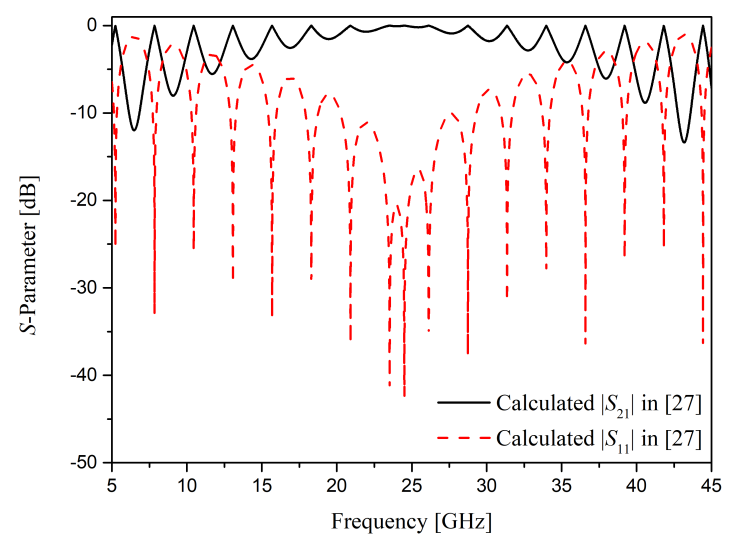

(a)

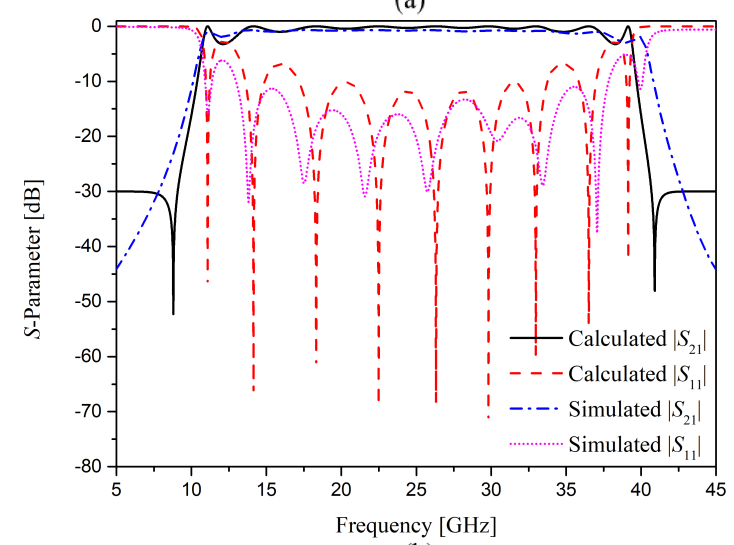

(b)

Fig. 4. Comparison of $S$-Parameter results among calculation based on method in [26], method in this paper and high frequency full wave simulation: (a) calculation based on the method in [26]; (b) calculation based on the method in this paper and simulation.

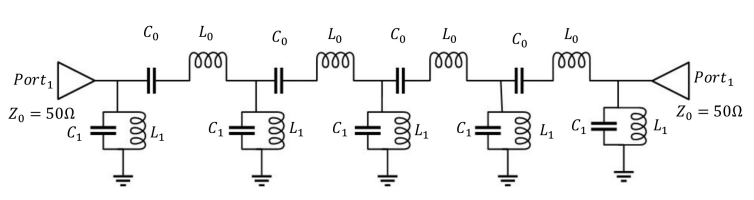

Fig. 5. The equivalent circuit model of the structure shown in Fig. 3.

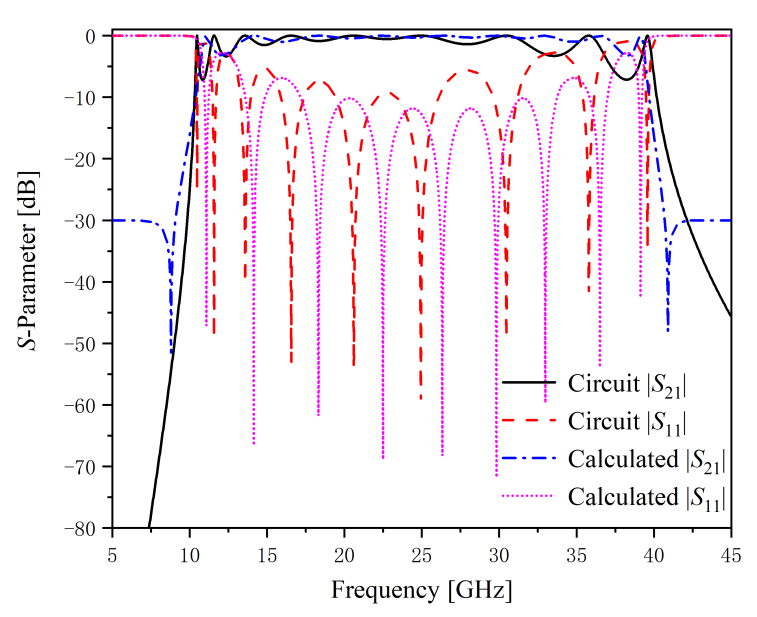

Fig. 6. Comparison of frequency responses between the equivalent circuit and the calculation method. 


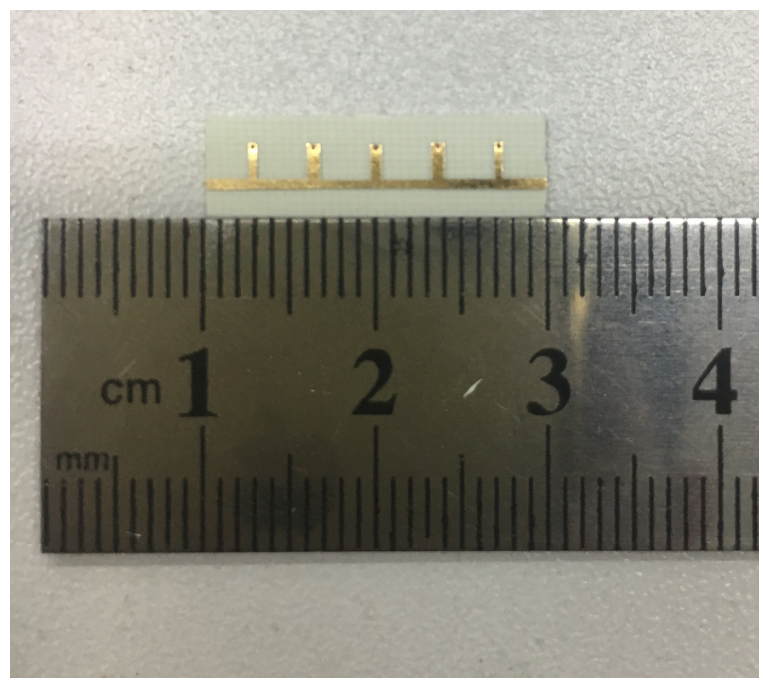

Fig. 7. The fabricated filter.

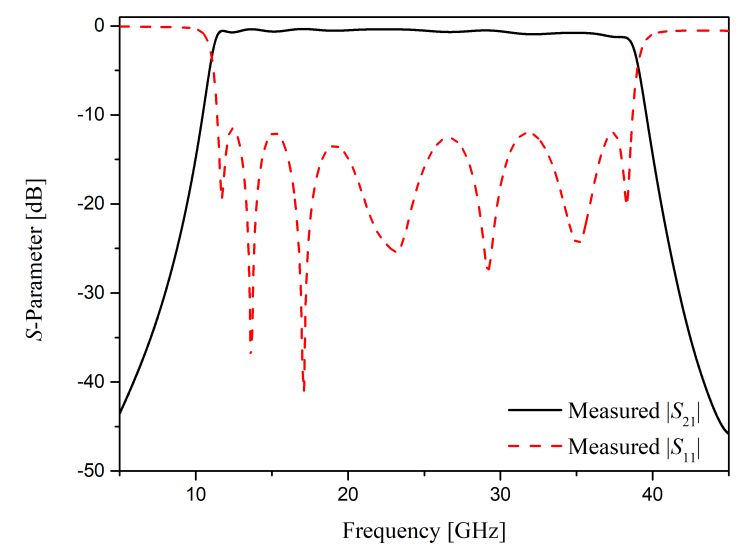

Fig. 8. Measurement results of the optimized filter.

\section{Calculation Procedure and Discussion}

\subsection{Reverse Calculating Procedure}

To obtain the dimensions of the microstrip filter by (9), the main goal is developing an objective function of the frequency responses and using some mathematical optimization method to find the optimal length of the stubs. In [26], the authors use the gradient descent method to search the optimal solutions of (4) (the optimal dimensions of the filter), but this method has some limitations. Because (4) is a nonconvex function, it could reach a local minimum in optimal searching, and the optimized results highly depend on the initialization. For the $\left|\Gamma_{\text {total }}\right|$ function proposed in this paper, it is a very complex function. Therefore, it is important for us to search a reliable solving method to reversely calculate the dimensions of the filter from $\left|\Gamma_{\text {total }}\right|$ function.

In this subsection, we propose a calculation procedure based on the Monte Carlo method. At the very beginning, we need to choose our interested frequency band and set the suitable optimization goals $F_{\text {goal }}$. A BPF is designed as an example. The optimization goals are shown in Tab. 5 .

\begin{tabular}{|l|l|l|l|}
\hline $\begin{array}{l}\text { Frequency Band } \\
{[\mathrm{GHz}]}\end{array}$ & Type of Band & Parameters & Goals \\
\hline $7-9.5$ & Passband & $\left|S_{11}\right|$ & $0.1(-10 \mathrm{~dB})$ \\
$5.5-6.5,10-11$ & Stop-band & $\left|S_{21}\right|$ & $0.01(-20 \mathrm{~dB})$ \\
\hline
\end{tabular}

Tab. 5. Optimization goals.

The dimensions of the filter are generated randomly and these generated values are put into $\left|\Gamma_{\text {total }}\right|$ function. The sampling interval of the frequency band is set as $10 \mathrm{MHz}$, and the calculated $\left|\Gamma_{\text {total }}\right|$ is compared with the optimization goals. Here we use the 2-norm difference as our objection function $\varepsilon$ :

$$
\begin{aligned}
\varepsilon_{\text {pass }}= & \left\|\mathbf{S}_{\mathbf{1 1}}-\mathbf{F}_{\text {goal }}\right\|_{2}^{2} \\
= & \sum\left[\left|S_{11}\right|\left(f_{n}\right)-F_{\text {goal }}\left(f_{n}\right)\right]^{2}, \\
\varepsilon_{\text {stop }}= & \left\|\mathbf{S}_{\mathbf{2 1}}-\mathbf{F}_{\text {goal }}\right\|_{2}^{2} \\
= & \sum\left[\left|S_{21}\right|\left(f_{n}\right)-F_{\text {goal }}\left(f_{n}\right)\right]^{2}, \\
& \varepsilon=\sqrt{\varepsilon_{\text {pass }}+\varepsilon_{\text {stop }} .}
\end{aligned}
$$

where $\varepsilon_{\text {pass }}$ represents the objection function in the passband, $\varepsilon_{\text {stop }}$ represents the objection function in the stop band, $f_{n}$ represents the $n$th sampling point of frequency, $\mathbf{S}_{11}$ represents the vector form of $\left|S_{11}\right|\left(f_{n}\right), \mathbf{S}_{21}$ represents the vector form of $\left|S_{21}\right|\left(f_{n}\right)$ and $\mathbf{F}_{\text {goal }}$ represents the vector form of $F_{\text {goal }}$.

For each calculation, the electrical lengths of the stubs are generated randomly, then those values are put into (9) and the difference between the $F_{\text {goal }}$ and the calculated curves obtained by (9) are compared. By executing the Monte Carlo experiments for $M$ times, the minimum value of $\varepsilon\left(\varepsilon_{\min }\right)$ and its corresponding dimensions of the stubs (the optimal electrical lengths) can be found. By these electrical lengths, the practical lengths of all stubs can be obtained simply. For the BPF being designed here, the relationship between $M$ and $\varepsilon_{\text {min }}$ is shown in Fig. 9.

As shown in Fig. 9, $\varepsilon_{\min }$ decreases with the increase of $M$, and $\varepsilon_{\text {min }}$ is fluctuating while $3000 \leqslant M \leqslant 15000$, but it is quite steady when $M$ is larger than 15000 . Therefore, we choose 15000 as the value of $M$ in the rest of this paper.

So far, the whole calculation procedure has been shown. The flowchart of this procedure is shown in Fig. 10, where $L^{(n)}$ represents the $n$th main line in Fig. 3 (from the left to the right). The value ranges of $N, L^{(n)}$ and $L_{x}^{(n)}$ are shown in Tab. 5. By using this procedure and the design goals in Tab. 5, here a bandpass filter is designed. The final $\varepsilon_{\min }$ is 0.8 . Although the error still exists, it is small enough to guide us to design the filter according the later results. 


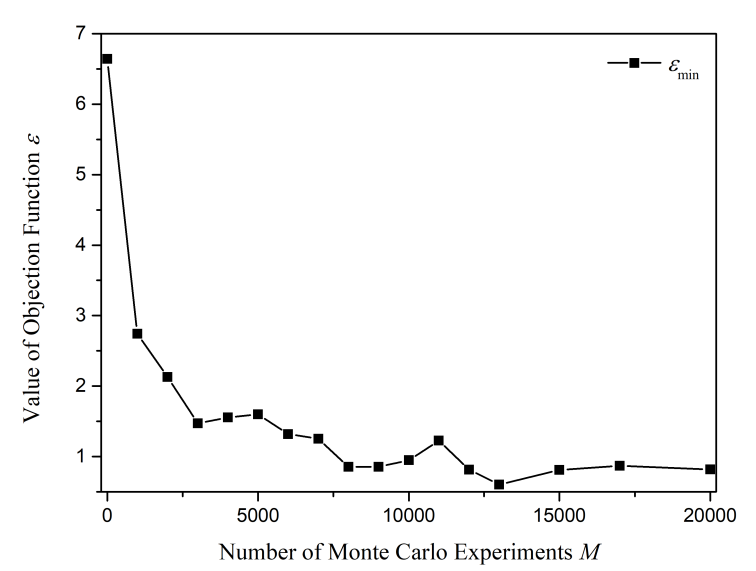

Fig. 9. Relationship between $M$ and $\varepsilon_{\min }$.

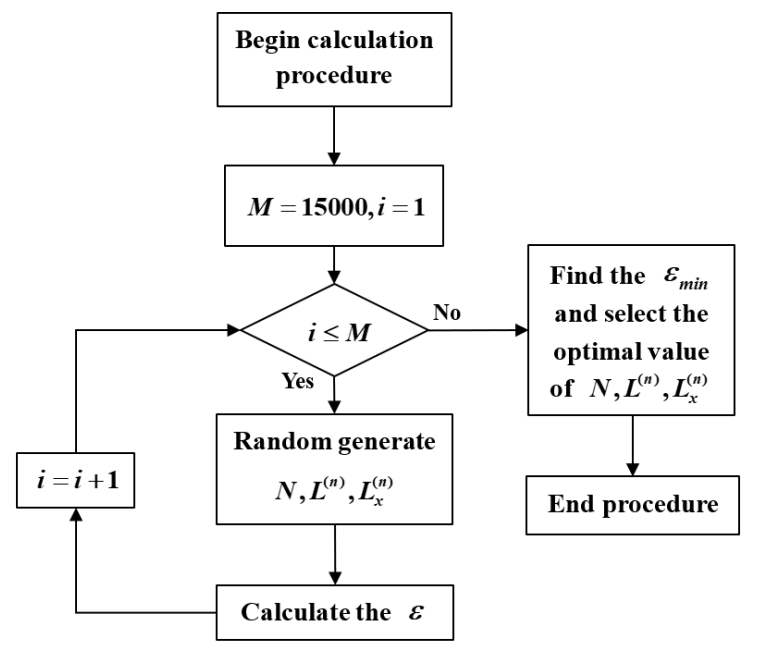

Fig. 10. Flowchart of the calculation procedure.

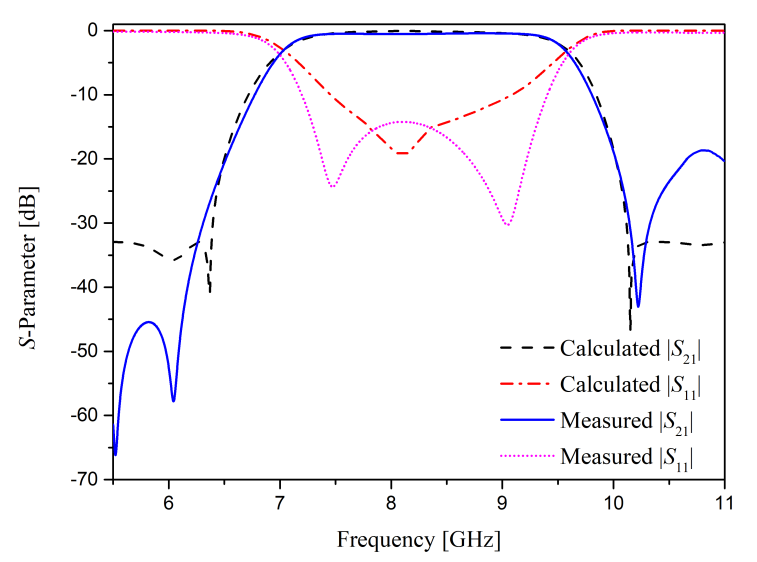

Fig. 11. $S$-parameter curves of the BPF.

\begin{tabular}{|l|l|l|l|}
\hline Parameter & $N$ & $L^{(n)}$ & $L_{x}^{(n)}$ \\
\hline Minimum Value & 3 & $0.5 \mathrm{~mm}$ & $0.5 \mathrm{~mm}$ \\
Maximum Value & 6 & $10 \mathrm{~mm}$ & $20 \mathrm{~mm}$ \\
\hline
\end{tabular}

Tab. 6. Value ranges of $N, L^{(n)}$ and $L_{x}^{(n)}$.
The comparisons between measured and calculated $S$-parameters are shown in Fig. 11. As it is depicted, the range of $\left|S_{11}\right|$ under $-10 \mathrm{~dB}$ is $7.1 \mathrm{GHz}-9.1 \mathrm{GHz}$. The ranges of $\left|S_{21}\right|$ under $-20 \mathrm{~dB}$ are $5.5 \mathrm{GHz}-6.5 \mathrm{GHz}$ and $10 \mathrm{GHz}-11 \mathrm{GHz}$. The calculated $S$-parameter results do not perfectly achieve the design goals, but it is enough to guide us in design process. Figure 11 also shows that the calculation results match the measurement results well. The calculated and practical dimensions of this BPF are shown in Tab. 7.

For this BPF, $L_{x}^{(2)}, L_{x}^{(4)}$ and $L_{x}^{(5)}$ are chosen as shortedstubs, and the other stubs are opened-stubs. Whether the stub is chosen to be opened- or shorted-stub can be achieved by setting a flag bit $x^{(n)}$ and we randomly generate it in the design procedure. For example, we can use this $x^{(n)}$ to create a binary detector: if $x^{(n)}$ equals to 1 , set the $L_{x}^{(n)}$ as an openedstub; if $x^{(n)}$ equals to 0 , set the $L_{x}^{(n)}$ as a shorted-stub.

As shown in Tab. 7, the differences between calculated and practical dimensions are very small, and it means that using our proposed method can save plenty of time while we optimize the filter in the EM software. The stub-loaded structure can be designed to a small size by bending its stubs and this is also a big advantage of our proposed microstrip structure. The fabricated bandpass filter is shown in Fig. 12. As the Fig. 12 shows, by bending the stubs, the space on the substrate board can be saved, and the size of the filter can be shaped into a small size.

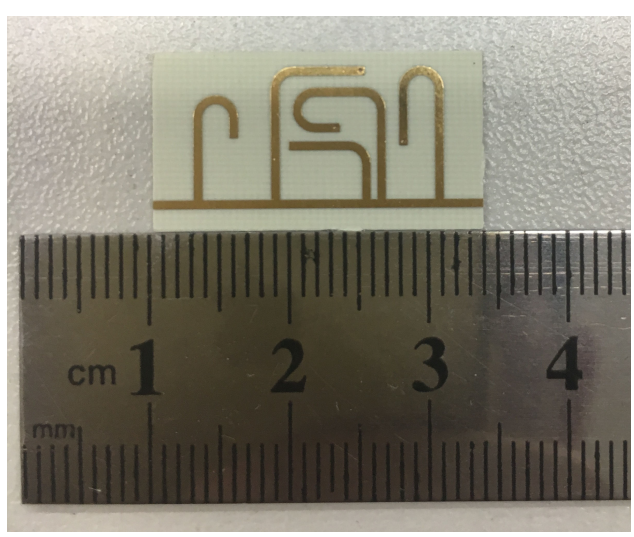

Fig. 12. The fabricated BPF.

\begin{tabular}{|l|l|ll|}
\hline Parameter & $\begin{array}{l}\text { Calculated Dimension } \\
{[\mathrm{mm}]}\end{array}$ & $\begin{array}{l}\text { Practical Dimension } \\
{[\mathrm{mm}]}\end{array}$ \\
\hline$L_{1}$ & 5 & 5 & \\
$L_{2}$ & 6 & 6 & \\
$L_{3}$ & 0.5 & 0.5 & \\
$L_{4}$ & 3.6 & 3.6 & \\
$L_{x}^{(1)}$ & 11.8 & 11.6 & \\
$L_{x}^{(2)}$ & 10.3 & 10 & \\
$L_{x}^{(3)}$ & 8.9 & 8.9 & \\
$L_{x}^{(4)}$ & 17.6 & 17.9 & \\
$L_{x}^{(5)}$ & 16.6 & 16.2 & \\
$W$ & - & 0.6 \\
\hline
\end{tabular}

Tab. 7. Calculated and practical dimensions of the bandpass filter. 


\subsection{Discussion}

\subsubsection{Comparison with Other Method}

Some properties and advantages of our proposed method are shown in this subsection.

Compared with the method in [26], our method can calculate more accurately. Furthermore, the method in this paper is less time-consuming compared with the method shown in [26]. The relationships among the number of iterations $M$, $\varepsilon_{\text {min }}$, and time cost are shown in Tab. 8. [26] use the logarithmic difference between (4) and goal function as the value of $\varepsilon_{\min }$, and we transform the $\varepsilon_{\min }$ into the same logarithmic form in Fig. 9. The calculations of both methods are done on the same computer.

Table 8 shows that our modified method can save plenty of calculation time compared with the method in [26]. The reason for this performance is that the gradient descent algorithm is used as optimal method in [26] and the computer need to calculate the partial derivative about (4) for dozens of times in each time of calculation. But for the method proposed in this paper, some further complex calculations are avoided, and we only need to calculate the difference between $\left|\Gamma_{\text {total }}\right|$ and the goal function each time. For the $\varepsilon_{\text {min }}$, the method based on the Monte Carlo experiments converges more quickly than the method based on the gradient descent algorithm. Therefore, the modified method proposed in this paper is more efficient than the method proposed in [26].

\begin{tabular}{|c|c|c|c|c|}
\hline \multirow{2}{*}{$\begin{array}{l}\text { Number } \\
\text { of } \\
\text { Iterations }\end{array}$} & \multicolumn{2}{|c|}{ Ref. [26] } & \multicolumn{2}{|c|}{ This Paper } \\
\hline & $\varepsilon_{\min }$ & time cost & $\varepsilon_{\min }$ & time cost \\
\hline 100 & 13.2 & $5 \mathrm{~s}$ & 18.3 & $0.6 \mathrm{~s}$ \\
\hline 4000 & 8.4 & $4 \mathrm{~min}$ & 6.8 & $9.5 \mathrm{~s}$ \\
\hline 8000 & 7.6 & $9 \min 10 \mathrm{~s}$ & 6.9 & $51.4 \mathrm{~s}$ \\
\hline 12000 & 7.2 & $14 \min 10 \mathrm{~s}$ & 4.1 & $1 \min 34 s$ \\
\hline 16000 & 5.7 & $21 \min 30 s$ & 3.3 & $2 \min 10 s$ \\
\hline 20000 & 4.7 & $29 \min 20 \mathrm{~s}$ & 3.2 & $3 \min 6 s$ \\
\hline
\end{tabular}

Tab. 8. Relationship among the number of iterations, $\boldsymbol{\varepsilon}_{\min }$ and time cost.

\begin{tabular}{|l|l|l|l|}
\hline & $\begin{array}{l}f_{\mathrm{c}}^{\text {(pass) }} \\
{[\mathrm{GHz}]}\end{array}$ & $f_{\mathrm{c}}^{\text {(stop) }[\mathrm{GHz}]}$ & $\begin{array}{l}\text { Length } \\
{[\mathrm{mm}]}\end{array}$ \\
\hline $\begin{array}{l}\text { Chebyshev BPF } \\
\text { Proposed BPF }\end{array}$ & $\begin{array}{l}6.7-9.9 \\
7-9.5\end{array}$ & $\begin{array}{l}5.5-6.2,10.2-11 \\
5.5-6.5,10-11\end{array}$ & 2.4 \\
\hline
\end{tabular}

Tab. 9. Comparison between Chebyshev BPF and the proposed BPF.

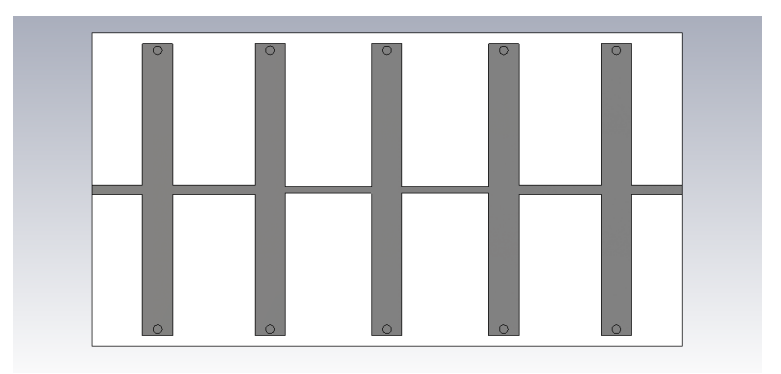

Fig. 13. Chebyshev BPF filter.

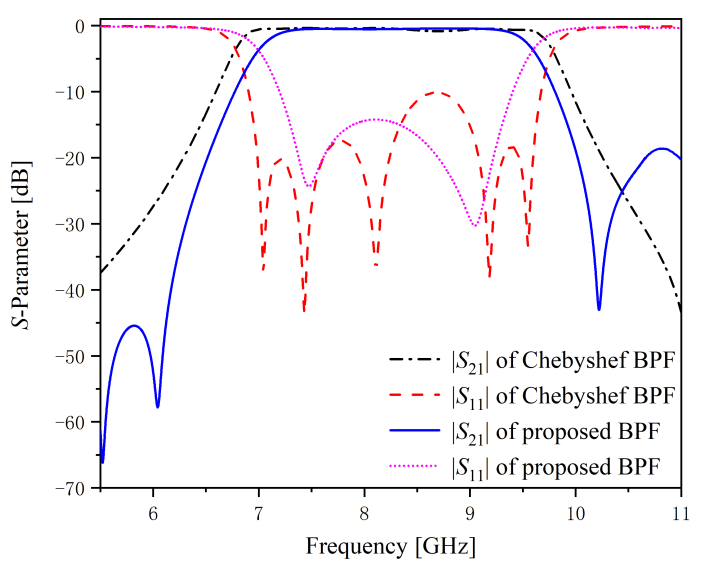

Fig. 14. Frequency responses of the Chebyshev and our proposed BPF filter.

As it is shown in this paper, a filter design method with a high-speed design process is proposed. Here we use the Chebyshev method proposed in [30] to redesign the BPF shown in Sec. 3.1 and compare its results with the results obtained by our proposed method. The detail of the design procedure is explained in [30], and we only give out the results. The structure of the filter based on Chebyshev method is shown in Fig. 13, and the frequency responses of this filter are shown in Fig. 14. As it is depicted in the Fig. 14, by using our method, we can design a filter which has the frequency responses as good as the Chebyshev filter has. The comparison between BPFs obtained by Chebyshev and our proposed methods is shown in Tab. 9, where $f_{\mathrm{c}}^{\text {(pass) }}$ represents the $3 \mathrm{~dB}$ passband cut-off frequency, and $f_{\mathrm{c}}^{\text {(stop) }}$ represents the $20 \mathrm{~dB}$ stop-band cut-off frequency.

But if we focus on the design procedure of the Chebyshev filter, we can find it is much more complex than our proposed method: Firstly, the order of equal-ripple low-pass filter prototype need to be selected while using Chebyshev method. Secondly, the capacitor values and the inductor values of the circuit mode need to be calculated according to the Chebyshev polynomials. Thirdly, the circuit of the LPF prototype need to be translated into a BPF circuit using the method proposed in [30]. Finally, the circuit mode need to be translated into microstrip line using the theory of Richards' transformation. As a comparison, by using the method proposed in this paper, the dimensions of the filter can be selected directly and automatically.

\subsubsection{Dimensions of the Filter}

As it is shown in Fig. 12, the structure of our proposed filter is an asymmetric structure. Compared with the symmetric structure proposed in [26], the asymmetric structure can prevent the waste of orders of the filter, and it is effective to miniaturize the shape of the filter by using the asymmetric structure. Let us consider a point symmetrical structure of the proposed BPF (shown in Fig. 15), where the order $N$ of the BPF equals to 10 . The $(N / 2-i)$ th sub and the $(N / 2+i+1)$ th stubs use the same type of loaded-stub, and their lengths equal to each other $(i=0,1, \cdots, N / 2-1)$. The symmetrical BPF also use the practical dimensions shown in Tab. 7. 
The frequency responses of the symmetric structure are simulated, and they are shown in Fig. 16. Compared with the asymmetric structure, the 10-order symmetric BPF can be regarded as a series connection of two asymmetric BPF. Therefore, the out-of-band rejection of the symmetric BPF can be better than the asymmetric BPF. But the passband can be narrower, and the insert loss in the passband can be worse.

As a conclusion, if the requirements of the filter performance are not extremely high, we can use the asymmetric structure to control the size of the filter.

Here we design a LPF based on the proposed method and compare this LPF with the filters designed in [24] and [29]. The prototype of our LPF is shown in Fig. 17 and its frequency response is shown in Fig. 18. All the parallel stubs we used are opened stubs. The calculated and practical dimensions of this filter are shown in Tab. 10. The comparisons of the $3 \mathrm{~dB}$ passband cut-off frequency $f_{\mathrm{c}}^{\text {(pass) }}$, $20 \mathrm{~dB}$ stop-band cut-off frequency $f_{\mathrm{c}}^{\text {(stop) }}$ and the lengths of filters are listed in Tab. 11.

\begin{tabular}{|l|l|l|}
\hline Parameter & $\begin{array}{l}\text { Calculated } \\
\text { Dimension }[\mathrm{mm}]\end{array}$ & $\begin{array}{l}\text { Practical } \\
\text { Dimension }[\mathrm{mm}]\end{array}$ \\
\hline$L_{1}$ & 3.9 & 3.3 \\
$L_{2}$ & 4.7 & 3.6 \\
$L_{3}$ & 4.9 & 4.9 \\
$L_{4}$ & 9.7 & 9.7 \\
$L_{x}^{(1)}$ & 8.6 & 8.6 \\
$L_{x}^{(2)}$ & 1 & 1 \\
$L_{x}^{(3)}$ & 6.3 & 6.6 \\
$L_{x}^{(4)}$ & 3.1 & 3.1 \\
$L_{x}^{(5)}$ & 0.6 & 0.6 \\
$W$ & - & 0.6 \\
\hline
\end{tabular}

Tab. 10. Calculated and practical dimensions of our low-pass filter.

\begin{tabular}{|l|l|l|l|}
\hline Characteristic & $\begin{array}{l}\text { Filter } \\
\text { in [28] }\end{array}$ & $\begin{array}{l}\text { Filter } \\
\text { in [29] }\end{array}$ & $\begin{array}{l}\text { Filter in this } \\
\text { Paper }\end{array}$ \\
\hline$f_{\mathrm{c}}^{\text {(pass) }}[\mathrm{GHz}]$ & $0-4$ & $0-3.5$ & $0-4$ \\
$f_{\mathrm{c}}^{\text {(stop) }[\mathrm{GHz}]}$ & 6 & 5 & 5 \\
Length $[\mathrm{mm}]$ & 14.3 & 2.4 & 2 \\
\hline
\end{tabular}

Tab. 11. Comparison among low-pass filters in [24], [29] and this paper.

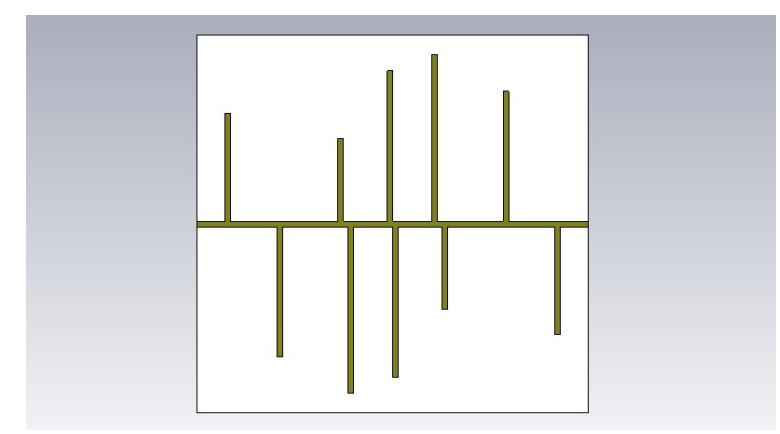

Fig. 15. Point symmetrical structure of the proposed BPF.

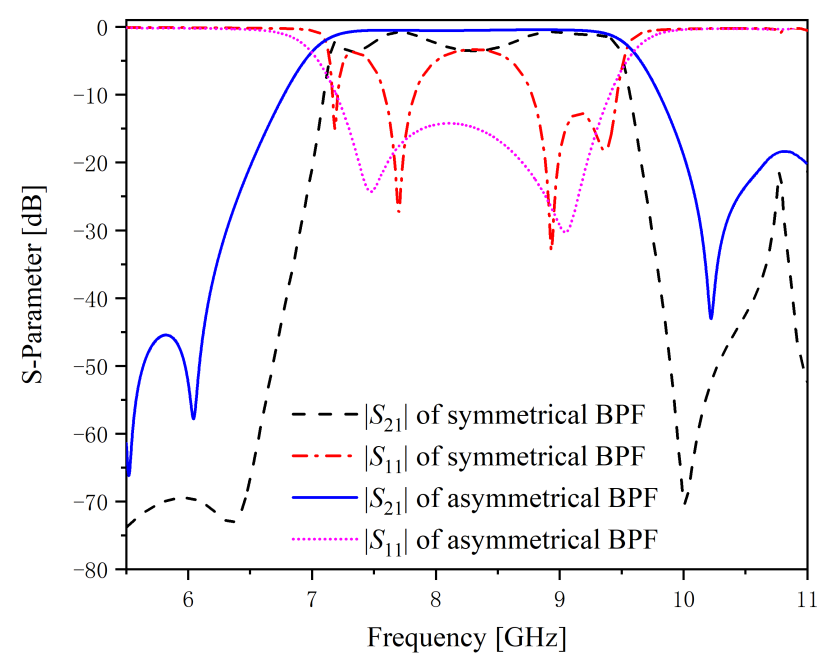

Fig. 16. Frequency responses of the symmetrical structure and the asymmetric structure of the BPF.

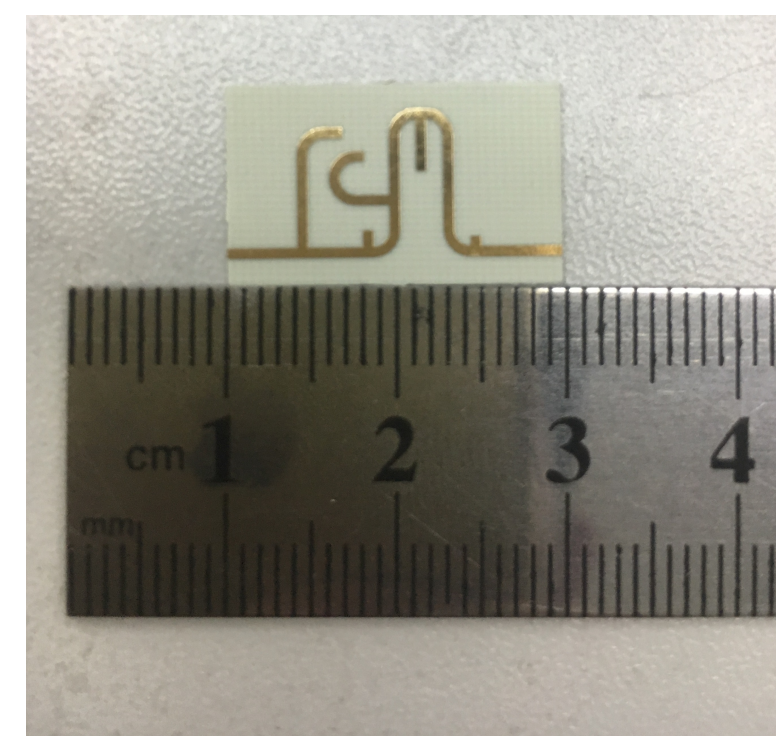

Fig. 17. The fabricated LPF.

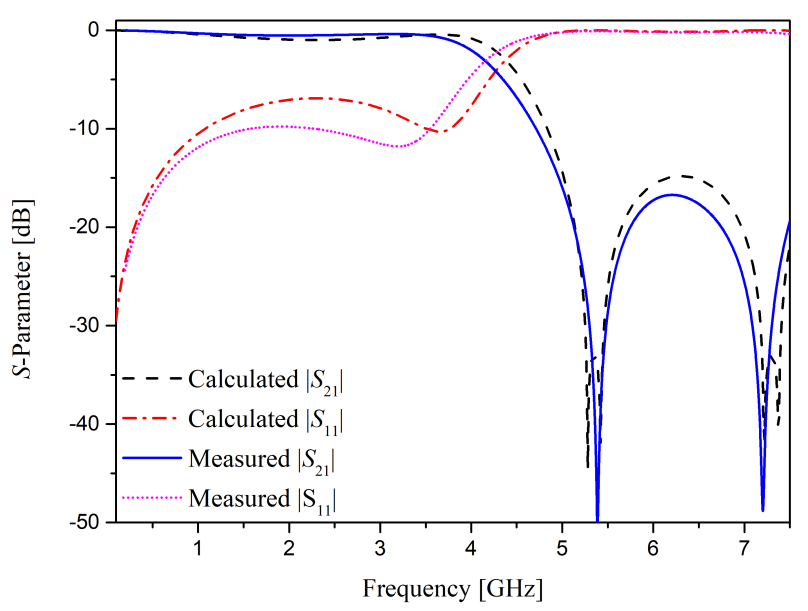

Fig. 18. $S$-parameter curves of the LPF. 
The filter designed in [24] is a stepped impedance filter based on the traditional Butterworth approximation method. The structure is simple but the length is around 7 times longer than our proposed filter, and its stop-band attenuation decays slowly. The filter shown in [29] is designed based on spiral compact microstrip resonant cells (NSCMRCs), and this filter has high performance in the stop-band and can be fabricated in small size. The structure of this filter is quite complex, so it is difficult to design and fabricate. Our filter eliminates the defects and contains the advantages mentioned above. Besides, our filter can perform well both in the passband and the stop-band and the size of our filter can be very small.

In [26], a dual-band filter with narrow passbands has been designed by using the reflection theory. And simulated result shows that the proposed method in [26] is effective under narrow band and multi-band situation. The dual-band filter proposed in [26] is shown in Fig. 19.

As a modified version of the method proposed in [26], the method proposed in our paper also can be used to design narrow band and multi-band filters. The parameters of the dual-band filter proposed in [26] are substituted into (9), and the frequency responses of this filter are calculated.

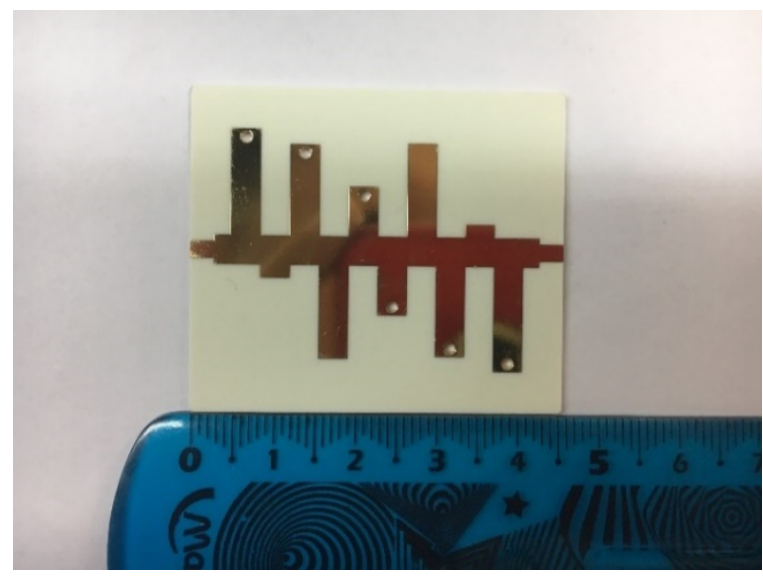

Fig. 19. Dual-band microstrip filter proposed in [26].

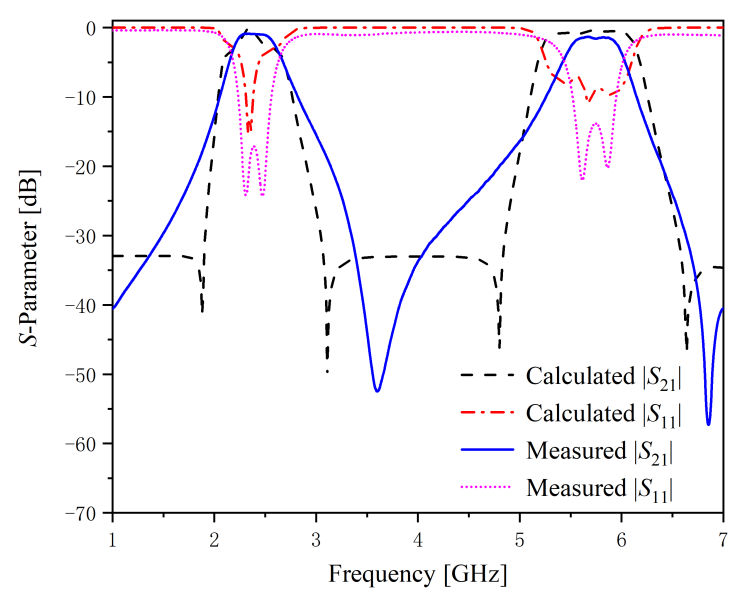

Fig. 20. Frequency responses of the dual-band filter proposed in [26].

\section{Conclusions}

In this paper, a novel method to design microstrip filters is proposed, and the method is based on the reflection theory and the calculation procedure of filter dimensions is based on the Monte Carlo experiments. The derivation procedure of $\left|\Gamma_{\text {total }}\right|$ is shown in Sec. 2 and the details of design procedure are shown in Sec. 3. Three filters are designed and fabricated. In Sec. 2.2, an UWB filter is designed to show that the calculation of $\left|\Gamma_{\text {total }}\right|$ is accurate; A bandpass filter is designed in Sec. 3.1 to show that our reverse calculating procedure is feasible; A low-pass filter is designed and compared with some prior arts in Sec. 3.2 to show the advantages of our proposed method and filter. All three above filters perform well, and the calculated and practical dimensions of stubs show a satisfactory fitting degree. At the end of Sec. 3, the frequency responses of a dual-band filter shows that our proposed method not only apply to the wide-band situation but also to the narrow band and multi-band situations. Besides, the design procedure of our method is simple and can calculate quickly compared with the method in [26]. The structure of designed filters is simple and their size is small by using our method. In conclusion, our method can be a good guidance in all kinds of microstrip filters design process.

\section{References}

[1] ANGUERA, J., ANDUJAR, A., HUYNH, M., et al. Advances in antenna technology for wireless handheld devices. International Journal on Antenna and Propagation, 2013, vol. 1921, p. 388-391. DOI:10.1155/2013/838364

[2] MA, P., WEI, B., HONG, J., et al. A design method of multimode multiband bandpass filters. IEEE Transactions on Microwave Theory \& Techniques, 2018, vol. 99, p. 2791-2799. DOI:10.1109/TMTT.2018.2815682

[3] CHEN, C.Y., HSU, C.Y. A simple and effective method for microstrip dual-band filters design. IEEE Microwave \& Wireless Components Letters, 2006, vol. 16, p. 246-248. DOI:10.1109/LMWC.2006.873584

[4] VAGNER, P., KASAL, M. A novel microstrip low-pass filter design method using square-shaped defected ground structure. Microwave \& Optical Technology Letters, 2010, vol. 50, p. 2458-2462.

[5] POZAR, D. M. Impedance matching and tuning. Microwave Engineering. $4^{\text {th }}$ ed. New Jersey, USA: John Wiley and Sons Inc., 2012, Ch. 5, Sec. 5, p. 250-252. ISBN:8126510498

[6] HENDIJANI, N., KHALAJ-AMIRHOSEINI, M., SHARGHI, V. K., et al. Design, simulation, and fabrication of tapered microstrip filters by applying the method of small reflections. In International Symposium on Telecommunications. Tehran (Iran), 2008, p. 133-137. DOI: 10.1109/ISTEL.2008.4651287

[7] POURMOHAMMADI, F., HAKKAK, M. Adaptive matching of antenna input impedance. In IEEE International Workshop Antenna Technology Small Antennas and Novel Metamaterials. White Plains (USA), 2006. DOI:10.1109/IWAT.2006.1609053

[8] SILVA, F. G. S., DE LIMA, R. N., FREIRE, R. C. S., et al. A switchless multiband impedance matching technique based on multirseonant circuits. IEEE Transactions on Circuits and System, 2017, vol. 59, no. 8, p. 1791-1800. DOI:10.1109/TCSII.2013.2261176.

[9] OLOKEDE, S. S., PAUL, B. S. A novel microstrip feed based on the theory of small reflection. In IEEE Radio and Antenna Days of the India Ocean. St. Gilles-les-Bains (Reunion), 2016. DOI:10.1109/RADIO.2016.7772018 
[10] MARTINS, G. S., SERDIJN, W. A. Multistage complex-impedance matching network analysis and optimization. IEEE Transactions on Circuits and System II-Express Briefs, 2016, vol. 63, no. 9, p. 833-837. DOI:10.1109/TCSII.2016.2534738

[11] ZENTNER, R., BARTOLIC, J., ZENTNER, E. Broadband matching of stacked patch antennas using a single line-transformer technique. Microwave and Optical Technology Lettters, 2003, vol. 39, no. 3, p. 178-183. DOI:10.1002/mop.11162

[12] El-HALABI, H., ISSA, H., PISTONO, E., et al. Compact lowpass stepped impedance filters wih enhanced out of band response. Microwave and Optical Technology Letters, 2017, vol. 59, no. 8, p. 1791-1800. DOI:10.1002/mop.30635

[13] GAN, D. C., HE, S. B., DAI, Z. J., et al. A quad-band bandpass filter using split-ring based on T-shaped stub-loaded stepped-impedance resonators. Microwave and Optical Technology Letters, 2017, vol. 59, no. 8, p. 2098-2104. DOI:10.1002/mop.30684

[14] CHIOU, Y. C., KUO, J. T. Compact UWB bandpass filter using stub-loaded multiple-mode resonator. IEEE Microwave and Wireless Components Letters, 2007, vol. 17, no. 11, p. 811-811. DOI:10.1109/LMWC.2007.908062

[15] KUO, J. T., SHIH, E. Microstrip stepped impedance resonator bandpass filter with an extended optimal rejection bandwidth. IEEE Transactions on Microwave Theory and Techniques, 2007, vol. 51, no. 5, p. 1554-1559. DOI:10.1109/TMTT.2003.810138

[16] DING, L., WANG, X. Z., ANG, N. S. S., et al. Ultrathin film broadband terahertz antireflection coating based on impedance matching method. IEEE Journal of Selected Topics in Quantum Electronics, 2017, vol. 23, no. 4, p. 1-8. DOI:10.1109/JSTQE.2016.2629666

[17] TORRUNGRUENG, D., THIMAPORN, C., LAMULTREE, S., et al. Theory of small reflections for conjugately characteristicimpedance transmission lines. IEEE Microwave and Wireless Components Lettters, 2008, vol. 18, no. 10, p. 659-661. DOI:10.1109/LMWC.2008.2003450

[18] KIM, C., SHRESTHA, B., SON, K.C. Wideband bandstop filter using an SIR based interdigital capacitor. Microwave and Optical Technology Letters, 2018, vol. 60, no. 10, p. 2530-2534. DOI: 10.1002/mop.31385

[19] WANG, X., WANG, J., CHOI, W., et al. Dual-wideband filtering power divider based on coupled stepped-impedance resonators. IEEE Microwave and Wireless Components Letters, 2018, vol. 28, no. 10, p. 873-875. DOI:10.1109/LMWC.2018.2861578

[20] CAI, J., YANG, Y., QIN, W., et al. Wideband tunable differential bandstop filter based on double-sided parallel-strip line. IEEE Transactions on Components, Packaging and Manufacturing Technology , 2018, vol. 8, no. 10, p. 1815-1822. DOI: 10.1109/TCPMT.2018.2794993

[21] KUMAR, L., PARIHAR, M. A wide stopband low-pass filter with high roll-off using stepped impedance resonators. IEEE Microwave and Wireless Components Letters , 2018, vol. 28, no. 5, p. 404-406. DOI: 10.1109/LMWC.2018.2816520

[22] LIU, H., LIU, F., GUANG, H., et al. Wide-stopband superconducting bandpass filter using slitted stepped-impedance resonator and composite spurline structure. IEEE Transactions on Applied Superconductivity, 2018, vol. 28, no. 8, DOI: 10.1109/TASC.2018.2858757

[23] LIU, H., SONG, Y., REN, B., et al. Balanced tri-band bandpass filter design using octo-section stepped-impedance ring resonator with open stubs. IEEE Microwave and Wireless Components Letters, 2017, vol. 27, no. 10, p. 404-406. DOI: 10.1109/LMWC.2017.2748340

[24] MAITY, B. Stepped impedance low pass filter using microstrip line for C-band wireless communication. In International Conference on Computer Communication and Informatics, Coimbatore (India), 2016, 4 p. DOI: $10.1002 /$ mop.31385

[25] DE ALENCAR, D. C., MENEZES, R.A.X. Direct synthesis of microwave filters using modified small reflection theory. International Microwave and Optoelectronics Conference, Rio de Janeiro (Brazil), 1999, p. 183-186. DOI:10.1109/IMOC.1999.867085
[26] YUAN, Y., WU, W., YUAN, W., et al. A method based on the theory of small reflections to design arbitrary passband microstrip filters. Radioengineering, 2018, vol. 27, no. 1, p. 214-220. DOI:10.13164/re.2018.0214

[27] MORIN, R. L. Monte Carlo simulation: A ubiquitous tool. Journal of the American College of Radiology, 2017, vol. 14, no. 3, p. 416-417. DOI:10.1016/j.jacr.2016.12.014

[28] BRAZALEZ, A. A., FlyGaRe, J., YANG, J., et al. Design of F-band transition from microstrip to ridge gap waveguide including monte carlo assembly tolerance analysis. IEEE Transactions on $\mathrm{Mi}$ crowave Theory and Techniques, 2016, vol. 64, no. 4, p. 1245-1254. DOI:10.1109/TMTT.2016.2535334

[29] HAYATI, M., SHEIKHI, A. Compact lowpass filter with ultrawide stopband using novel sprial compact microstrip resonant cell. IEICE Electronics Express, 2011, vol. 8, no. 13, p. 1028-1033. DOI: $10.1587 /$ elex.8.1028

[30] DONG, X. M., ZHOU, D. X. Learning gradients by a gradient descent algorithm. Journal of Mathematical Analysis and Applications, 2008, vol. 341, no. 2, p. 1018-1027. DOI:10.1016/j.jmaa.2007.10.044

\section{About the Authors ...}

Yuhong MA was born in 1996. He received his B.S. degree from National University of Defense Technology in 2017. Currently he is working towards the M.S. degree in the State Key Laboratory of Complex Electromagnetic Environment Effects on Electronics and Information System, National University of Defense Technology. His research interests include wireless communication and wave propagation.

Ye YUAN (Corresponding author) was born in 1994. He received his B.S. degree in Electronic Science and Technology from the Nanjing University of Aeronautics and Astronautics in 2016. Currently he is working towards the M.S. degree in the College of Electronic Science and Engineering, National University of Defense Technology, Changsha, Hunan, China. His research interests include passive microwave circuits design and wireless communication.

Wentao YUAN was born in 1990. He received his B.S. degree in Computer Science and Technology from the Anhui Normal University in 2016. Currently he is working towards the M.S. degree in the College of Electronic Science and Engineering, National University of Defense Technology, Changsha, Hunan, China. His research interests include passive microwave circuits design and wireless communication.

Weiwei WU was born in 1981. She received her M.S. and Ph.D. degree in Electronic Science and Technology from the National University of Defense Technology in 2008 and 2011, respectively. Currently she is a teacher in the College of Electronic Science and Engineering, National University of Defense Technology, Changsha, Hunan, China. Her research interests include antennas design and wave propagation.

Naichang YUAN was born in 1965. He received his M.S. and Ph.D. degree in Electronic Science and Technology from the University Science and Technology of China in 1991 and 1994, respectively. He is currently a professor with the College of Electronic Science and Engineering, National University of Defense Technology, Changsha, Hunan, China. His research interests include microwave circuits design, wireless communication and wave propagation. 